\title{
Kinetic Monte Carlo Modelling of Charge Carriers \\ in Organic Electronic Devices: Suppression of the Self-Interaction Error
}

\author{
Haoyuan $\mathrm{Li}^{\dagger}$ and Jean-Luc Brédas ${ }^{\dagger} *$ \\ Laboratory for Computational and Theoretical Chemistry of Advanced Materials \\ Physical Science and Engineering Division \\ King Abdullah University of Science and Technology \\ Thuwal 23955-6900, Kingdom of Saudi Arabia
}

\section{Corresponding Author}

* jean-luc.bredas@chemistry.gatech.edu

${ }^{\dagger}$ New permanent address: School of Chemistry and Biochemistry, Center for Organic Photonics and Electronics (COPE), Georgia Institute of Technology, Atlanta, Georgia 30332-0400, United States 


\section{ABSTRACT}

Kinetic Monte Carlo (KMC) simulations have emerged as an important tool to help improve the efficiency of organic electronic devices by providing a better understanding of their device physics. In the KMC simulation of an organic device, the reliability of the results depends critically on the accuracy of the chosen charge-transfer rates, which are themselves strongly influenced by the site-energy differences. These site-energy differences include components coming from the electrostatic forces present in the system, which are often evaluated through electric potentials described by the Poisson equation. Here, we show that the charge-carrier selfinteraction errors that appears when evaluating the site-energy differences, can lead to unreliable simulation results. To eliminate these errors, we propose two approaches that are also found to reduce the impact of finite-size effects. As a consequence, reliable results can be obtained at reduced computational costs. The proposed methodologies can be extended to other device simulation techniques as well.

\section{TOC GRAPHICS}

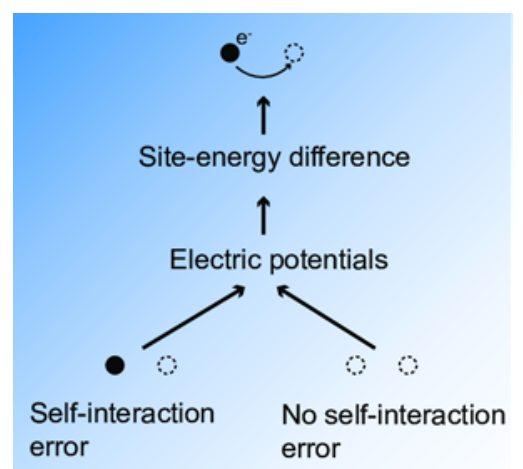


KEYWORDS: organic electronic devices, kinetic Monte Carlo simulations, charge mobility, charge transport, Poisson’s equation. 
Organic electronic devices are promising light-weight, flexible devices for low-cost applications. ${ }^{1-4}$ However, to take full advantage of these attractive features and have such devices integrated into consumer products, further improvements in performance and lifetime are needed. A deeper understanding of the microscopic processes taking place upon operation of the devices can go a long way in achieving these goals. In this context, kinetic Monte Carlo (KMC) simulations have emerged as a very useful tool ${ }^{5-11}$ and have been applied to the description of organic solar cells (OSCs) ${ }^{12-17}$, organic light-emitting diodes (OLEDs) ${ }^{18-21}$, and organic fieldeffect transistors (OFETs) ${ }^{22-26}$. In order for such simulations to play a useful role in improving our comprehension of the device physics and efficiencies, it is critical that the KMC results are reliable.

KMC simulations describe the time evolution of the microscopic processes taking place upon device operation. Therefore, the accuracy obtained for the rates of these processes largely determines the accuracy of the KMC simulations themselves. In organic electronic devices, a key process is the motion of the charge carriers. For the vast majority of organic molecular semiconductors, such motions can be depicted as hops of the charges among neighboring molecules ${ }^{27,28}$; the associated rates for hops between molecule $i$ and molecule $j$ are usually described by either the Miller-Abrahams equation ${ }^{29}$ or the Marcus equation ${ }^{30}$ :

$$
\begin{aligned}
& k_{M A}=v_{0} \exp \left(-2 \gamma\left|R_{i j}\right|\right)\left\{\begin{array}{cc}
\exp \left(-\frac{E_{j}-E_{i}}{k_{B} T}\right) & E_{j}>E_{i} \\
1 & E_{j} \leq E_{i}
\end{array}\right. \\
& k_{\text {Marcus }}=\frac{2 \pi^{2} t^{2}}{h \sqrt{\pi \lambda k_{B} T}} \exp \left(-\frac{\left(E_{j}-E_{i}+\lambda\right)^{2}}{4 \lambda k_{B} T}\right)
\end{aligned}
$$


where $v_{0}$ denotes the effective (phonon) vibration frequency; $\gamma$, the inverse localization radius; $R_{i j}$, the distance from site $i$ to $j ; E_{i}$ and $E_{j}$, the site energies; $T$, the temperature; $k_{B}$, the Boltzmann constant; $h$, the Planck constant; $\lambda$, the reorganization energy; and $t$, the charge transfer integral. Since the site-energy difference $\left(E_{j}-E_{i}\right)$ appears in the argument of an exponential term, its accuracy will have a strong influence on the accuracy of the calculated charge-transfer rates, which in turn greatly impact the actual simulation results.

The site-energy difference has an "internal" component coming from the intrinsic energy levels of the molecules $\left(E_{i n}\right)$ in the solid and an "external" component coming from the electric field $\left(E_{e x}\right):^{9}$

$$
E_{j}-E_{i}=\left(E_{i n, j}-E_{i n, i}\right)+\left(E_{e x, j}-E_{e x, i}\right)
$$

Multiple charge carriers need to be considered when simulating the device and the electric potential within the device at any given time can be described by the Poisson equation. The electric potential is usually re-evaluated at every simulation step since it evolves with the carrier motion. The electric potential values obtained for a given distribution of the charges (at a given voltage) have been used to evaluate directly the external component $E_{e x, j}-E_{e x, i}{ }^{9,21,22,31}$

$$
E_{e x, j}-E_{e x, i}=q\left(\varphi_{j}-\varphi_{i}\right)
$$

where $q$ is the charge of the carrier and $\varphi_{i}$ and $\varphi_{j}$ denote the electric potential at sites $i$ and $j$.

However, this procedure -based on the distribution of charges prior to a given hop- neglects the fact that the electric potential changes in the course of the hopping process. (We note that, while KMC simulations mainly consider the motions of charge carriers, they do not necessarily imply 
that other properties of the system remain unchanged). The use of Equation 4 does not account for the image-charge forces ${ }^{32,33}$ and includes an error due to carrier self-interactions. While the former issue can be alleviated by explicitly considering the image charges ${ }^{9}$, the self-interaction error has remained unaddressed. In this Letter, we discuss how it is possible to better evaluate the site-energy difference component in order to suppress the self-interaction error in KMC simulations of charge transport in organic electronic devices.

For the sake of simplicity, we define Equation $\mathbf{4}$ as corresponding to the "raw" method, where the electric potential for the initial configuration is directly used to calculate the external part of the site-energy difference. To properly take into account the evolution of the electric potential and suppress the carrier self-interaction error, the electric potential corresponding to the final carrier occupation can be explicitly considered. More specifically, the Poisson equation needs to be solved when considering every possible hopping process. We denote this procedure as the "exact" method (where the "exact" terminology has to be taken relative to the other methods discussed here).

At first sight, the computational cost could be expected to be extremely high. If we use $n$ and $k$ to denote the number of carriers and the number of allowed neighbors for a hopping process, respectively, the Poisson equation would need to be solved up to $n k+1$ times at each KMC step. Here, we propose a simple, yet very efficient, approach to achieve the same outcome while eliminating the need to solve the Poisson equation multiple times at each KMC step, where the energy difference due to the electric field writes as:

$$
E_{e x, j}-E_{e x, i}=q\left[\left(\varphi_{j}-\varphi_{j}^{(i)}+\varphi_{j}^{(j)}\right)-\left(\varphi_{i}\right)\right]
$$


where $\varphi_{j}^{(i)}$ denotes the electric potential at site $j$ due to the presence of a single carrier at site $i$, which is obtained by solving Poisson's equation for a single-carrier system (under the same boundary conditions with the boundary values set to 0$) ;{ }^{26} \varphi_{j}^{(j)}$ denotes the electric potential at site $j$ due to a single carrier at site $j$.

In order to suppress the self-interaction error, it is also useful to look at another case, where the site-energy difference coming from the electric field is evaluated from the electric-potential difference in the absence of the hopping carrier. This procedure would reduce the error associated with the "raw" method, while eliminating the need to consider two different carrier configurations for a hopping process. We denote this procedure as the "exclusion" method. In principle, this method requires solving the Poisson equation $n$ times at every KMC step. In a way similar to the approach adopted for the "exact" method, an alternative solution can be used to reduce the computational cost:

$$
E_{e x, j}-E_{e x, i}=q\left[\left(\varphi_{j}-\varphi_{j}^{(i)}\right)-\left(\varphi_{i}-\varphi_{i}^{(i)}\right)\right]
$$

where $\varphi_{i}{ }^{(i)}$ denotes the electric potential at site $i$ due to the presence of a single carrier on this site.

Because $\varphi_{i}^{(i)}, \varphi_{j}^{(i)}$ and $\varphi_{j}^{\left({ }^{(j)}\right.}$ can be obtained and stored prior to running the full KMC simulation, the increased computational cost of the "exact" or "exclusion" method is minimal, as it only requires the additional operation of retrieving two variables from memory when evaluating the site-energy difference for a hopping process. In our benchmark calculations, we find an increase in the overall simulation time by only about 4\%-10\% compared to the "raw" method (see Figure S1). 
The comparison of the three methods to calculate the site-energy difference from the electric field is shown in Figure 1a. We use two different devices in order to illustrate the various approaches: (i) a diode (Figure 1b); and (ii) an OFET (Figure 1c). The KMC simulation process follows the same path as in our previous works; ${ }^{26,34,35}$ thus, we only briefly recall it here. Each simulated device has a dimension of $L_{x} \times L_{y} \times L_{z}$. Different components of the devices (electrodes, organic semiconductors, and insulators) are represented by lattice sites in threedimensional space $\left(n_{x} \times n_{y} \times n_{z}\right)$; the site spacing is $1 \mathrm{~nm}$. For the diode, $n_{x}=n_{y}=50, n_{z}=101$; periodic boundary conditions (PBCs) are applied in both $x$ and $y$ directions; the [z=1] and $[z=101]$ sites correspond to the electrodes; the other sites represent the organic semiconductor. For the OFET device, $n_{x}=50, n_{y}=17, n_{z}=257$; PBCs are applied in the $x$ direction; the regions [9 $\leq y \leq 16, z=1],[9 \leq y \leq 16, z=257]$ and $[y=1]$ represent the source, drain, and gate electrodes, respectively; the channel length $(L)$ and the insulator dielectric thickness $(d)$ are $256 \mathrm{~nm}$ and 8 nm, respectively.

The site energy is assumed to have a Gaussian distribution typical of organic semiconductors; the density of electronic states (DOS), $g(E)$, writes as: ${ }^{5}$

$g(E)=\frac{1}{\sqrt{2 \pi \sigma^{2}}} \exp \left(-\frac{E^{2}}{2 \sigma^{2}}\right)$

where $\sigma$ is the energetic disorder. Typical values of the energetic disorder in organic semiconductors are in the range of 50-150 meV. ${ }^{36,37}$ In this study, we vary $\sigma$ between 51 and $128 \mathrm{meV}$, which is chosen to correspond to $2 k_{B} T-5 k_{B} T$ range at room temperature. We note that the charge carriers are forbidden to hop into the sites representing the dielectric and that no injection barrier is considered. The rates for charge transport (here, we deal with hole transport) and injection/collection are both described by the Miller-Abrahams equation. ${ }^{38}$ 
The diode has a sandwich structure, and the charge carriers mainly drift in the direction perpendicular to the electrode plane. If the electric-potential difference along this plane is neglected, the electric potential in the device can be described by the 1D Poisson equation: ${ }^{9}$

$$
\frac{\partial^{2} \varphi}{\partial z^{2}}=-\frac{\rho}{\varepsilon_{r} \varepsilon_{0}}
$$

where $\rho$ represents the charge density; $\varepsilon_{0}$, the vacuum permittivity; and $\varepsilon_{r}$, the relative permittivity; the latter is set to 4 , a value often found in organic materials. ${ }^{39}$

On the other hand, the OFET device has a pseudo-two-dimensional (pseudo-2D) structure, and its components remain uniform in the direction normal to the 2D plane. The electric potential in the device can be described using the 2D Poisson equation: ${ }^{26}$

$$
\frac{\partial^{2} \varphi}{\partial y^{2}}+\frac{\partial^{2} \varphi}{\partial z^{2}}=-\frac{\rho}{\varepsilon_{r} \varepsilon_{0}}
$$

All the calculated current values are based on the average of at least 16 simulations to obtain a converged result.

Figure 2 illustrates that, for a diode device, the currents derived from all three methodologies are similar. However, the OFET case is very different. As can be seen in Figure 3, the transfer currents estimated via the "raw" method are significantly higher than those derived with the other two methods; this difference is most prominent at high energetic disorders. On the other hand, the results obtained with the "exact" and "exclusion" methods remain very similar. The same trends are also found for the output currents (see Figure S2 in the Supplementary Information, SI). 
The differences observed in the transfer and output currents are mainly caused by the selfinteraction error of the "raw" method. Because Poisson's equation gives a lower electric potential at a site adjacent to a hole (Figure S3), the energy barrier for the hopping process is lowered. However, this lowered barrier is artificial, since its origin is related to the hopping carrier itself. Thus, the "raw" method leads to increased carrier motions and overestimated currents. For the OFET case, the error in the currents can be as large as $400 \%$ (Figure 3d), within our investigated parameter range. On the other hand, neither the "exact" method nor the "exclusion" method includes this error when evaluating the charge-transfer rates; it is therefore reasonable that the results using these two methods are close to each other, and are more reliable.

We have identified three factors that influence the extent of the self-interaction error:

(i) The dimensionality of the Poisson equation. The electric potential along the $z$ direction in the devices when a single charge carrier is present can be found in Figure S3. Within the investigated parameters, the electric-potential difference between adjacent sites can be as large as $38 \mathrm{mV}$ for the case of the 2D Poisson equation, while the largest value for the case of the 1D Poisson equation is only $1.5 \mathrm{mV}$. This explains the reason why the "raw" method results in larger errors in the OFET case than in the diode case.

(ii) The size of the system. The potential difference between the site where the hopping carrier is located and an adjacent site also depends on the system dimension perpendicular to the electric field. When the 1D [or 2D] Poisson equation is used, the carrier density is layer-averaged [or line-averaged]. As a result, when reducing $n_{x}$, this electric-potential difference (Figure S3a and S3b) increases. The error in the "raw" method is, therefore, expected to be larger in the case of smaller device dimensions. Indeed, significant differences in the currents appear for the three 
methods, even for the diode device, when reducing both $n_{x}$ and $n_{y}$ (Figure 4a). On the other hand, when increasing the channel width $(W)$ for the OFET device, the differences in the currents evaluated using the three methods become smaller (Figure 4b).

(iii) The effective energetic disorder. For materials with high energetic disorders, the carriers are essentially trapped at low-energy sites and the (artificial) enhancement on the carrier motion from the "raw" method is then also larger. Thus, this explains the larger error derived with the "raw” method at higher energetic disorders (Figures 3 and S2). As carriers fill the low-energy levels, a higher carrier density, which can be the result of an increased gate voltage, leads to decreased effective energetic disorder; therefore, the error due to the "raw" method is larger at lower gate voltages (Figure 3).

For the "raw" method, the currents have been found to increase at smaller device dimensions. Such a phenomenon was usually rationalized in terms of finite-size effects as insufficient sampling leads to reduced apparent energetic disorder; this, in turn, results in more efficient charge transport. However, single-carrier calculations show that within the investigated parameters, $n_{x}$ and $n_{y}$ have little influence over the charge mobilities (Figure S4). The implication is that the system size here is large enough for sufficient sampling. Thus, decreasing the system size does not necessarily lead to increased currents (or at least this tendency is reduced) when the self-interaction error is suppressed (Figures $\mathbf{4 a}$ and $\mathbf{4 b}$ ). These results suggest that the self-interaction error can be an important reason producing overestimated currents in device modelling, which can be corrected using the "exact” or "exclusion" method (Figures 4a and $\mathbf{4 b}$ ). Therefore, reliable results can be obtained at reduced system sizes, which translates into more affordable computational costs, since the KMC simulation time depends significantly on the system size. 
Interestingly, the currents from the "exact" method are found to increase when first increasing the system size (Figures $\mathbf{4 a}$ and $\mathbf{4 b}$ ). One reason can be understood as follows: The Poisson equation predicts that when charge carriers move through the device, they first experience an elevated energy barrier, then a reduced one (see Figure 4c). Since the carrier motion in the first part of the device is usually the limiting process due to the non-uniform electric field, ${ }^{26,34,40}$ the elevated energy barrier also leads to slower carrier motion. When reducing the device dimensions, these effects are more significant (Figure 4c), which leads to decreased currents. On the other hand, this energy barrier is absent in the "exclusion" method, which is why the results from this method are higher than those using the "exact" method. It is thus reasonable that larger differences are found between these two methods at small device dimensions (Figures $4 \mathbf{a}$ and 4b) and when the electric field in the OFET device is weak at low gate voltages (Figure 3).

In our discussion, we have considered the 1D and 2D Poisson equations in order to illustrate our methodologies in a simple manner. In such simulations, the short-range electrostatic forces are not fully taken into account and the 3D Poisson equation can be used to achieve a more accurate description of the electric potentials in the device. We note that the electric potential in diode configurations has been considered in KMC simulations by using a hybrid approach: ${ }^{9}$ shortrange electrostatic interactions are explicitly evaluated using Coulomb’s law, while long-range interactions are calculated in a layer-averaged way. In this instance, however, abrupt changes in the electric potential at the cutoff positions can result in fluctuations that influence the accuracy of the simulations. The way to reduce this error is to use a large cutoff, which, however, greatly increases the calculation cost. Based on the methodology proposed in this Letter, both shortrange and long-range electrostatic potentials can be consistently described using the 3D Poisson equation while suppressing the self-interaction error (see Figure S5a). 
Conceptually, for a diode-structure device, when the 3D Poisson equation is used, the "exact" method is similar to the hybrid approach with explicit consideration of image-charge forces. ${ }^{9}$ However, we note a difference when considering the image charges: the Poisson solver uses the potential value at the electrodes as the reference, while when considering image-charge forces, the reference is taken for a charge infinitely away from the electrodes; as a result, the electric potentials at the electrode/organic semiconductor interface derived from the 3D Poisson equation using the "exact" method, are different from those usually evaluated in the presence of imagecharge forces (see Figure S5b). On the other hand, the image-charge forces can still be explicitly considered when the "exclusion" method is used. In such a case, the simulation results can actually be close to those obtained using the hybrid approach with a large cutoff in the presence of image-charge forces (see Figure S6).

We would like to point out that the self-interaction error is also present in device simulations using techniques other than KMC (for example, the master equation approach ${ }^{41}$ ). To suppress the self-interaction error in such device modellings, the "exclusion" method can be generalized by incorporating the occupancies of charge carriers into Eq. (6):

$$
E_{e x, j}-E_{e x, i}=q\left[\left(\varphi_{j}-\beta_{i} \varphi_{j}^{(i)}-\beta_{j} \varphi_{j}^{(j)}\right)-\left(\varphi_{i}-\beta_{i} \varphi_{i}^{(i)}-\beta_{j} \varphi_{i}^{(j)}\right)\right]
$$

where $\beta_{i}$ and $\beta_{j}$ are the occupancies of charges at sites $i$ and $j$, respectively.

In conclusion, we have shown that the carrier self-interaction error present in the evaluation of the site-energy differences can result in substantial inaccuracies in kinetic Monte Carlo simulations of organic electronic devices. The extent of this error depends on several aspects: (i) the dimensionality of Poisson's equation; (ii) the system size; and (iii) the energetic disorder 
present in the system. Within the parameter range investigated in this work, inaccuracies in terms of current can be as large as $400 \%$. To eliminate this error, two methods have been proposed: the "exact" method, which takes into account both the initial and final carrier distributions / configurations, and the "exclusion" method, where the electric potentials are evaluated in the absence of the hopping carrier. These two approaches have been shown to increase the accuracy of the results and to reduce the finite-size effects in the simulations of diodes and organic fieldeffect transistors, which allows reliable results to be obtained at reduced computational costs. Based on the methodologies developed in this Letter, consistent descriptions of both the shortand long-range electric potentials can be achieved using the 3D Poisson equation while suppressing the self-interaction error. Importantly, the "exclusion" method can be generalized for device modelling using techniques other than KMC such as the master equation approach.

\section{ASSOCIATED CONTENT}

Supporting Information. The Supporting Information is available free of charge on the ACS Publications website. The SI includes: benchmarks of the simulation times for the "raw", "exclusion" and "exact" methods; calculated output currents for the OFET device; electric potentials from a single charge by the Poisson equation; mobilities from single-carrier simulations; electric potentials in the device obtained with the 3D Poisson equation or a hybrid approach; and calculated currents using the 3D Poisson equation compared to those using the hybrid approach.

\section{AUTHOR INFORMATION}




\section{Corresponding Author}

*Email: jean-luc.bredas@chemistry.gatech.edu

\section{Notes}

The authors declare no competing financial interests.

\section{ACKNOWLEDGMENTS}

This work was supported by internal funding from King Abdullah University of Science and Technology. We are grateful to the KAUST IT Research Computing Team and Supercomputing Laboratory for providing outstanding assistance as well as computational and storage resources.

\section{REFERENCES}

(1) D'Andrade, B. W.; Forrest, S. R. White Organic Light-Emitting Devices for Solid-State Lighting. Adv. Mater. 2004, 16, 1585-1595.

(2) Mishra, A.; Bauerle, P. Small Molecule Organic Semiconductors on the Move: Promises for Future Solar Energy Technology. Angew. Chem., Int. Ed. Engl. 2012, 51, 2020-2067.

(3) Myers, J. D.; Xue, J. G. Organic Semiconductors and Their Applications in Photovoltaic Devices. Polym. Rev. (Philadelphia, PA, U. S.) 2012, 52, 1-37.

(4) Sirringhaus, H. 25th Anniversary Article: Organic Field-Effect Transistors: The Path Beyond Amorphous Silicon. Adv. Mater. 2014, 26, 1319-1335.

(5) Bassler, H. Charge Transport in Disordered Organic Photoconductors - a Monte-Carlo Simulation Study. Physica Status Solidi B-Basic Research 1993, 175, 15-56.

(6) Houili, H.; Tutis, E.; Batistic, I.; Zuppiroli, L. Investigation of the Charge Transport through Disordered Organic Molecular Heterojunctions. J. Appl. Phys. 2006, 100, 033702. 
(7) Zhou, J.; Zhou, Y. C.; Zhao, J. M.; Wu, C. Q.; Ding, X. M.; Hou, X. Y. Carrier Density Dependence of Mobility in Organic Solids: A Monte Carlo Simulation. Phys. Rev. B 2007, 75, 153201.

(8) Novikov, S. V.; Vannikov, A. V. Hopping Charge Transport in Disordered Organic Materials: Where Is the Disorder? J. Phys. Chem. C 2009, 113, 2532-2540.

(9) van der Holst, J. J. M.; van Oost, F. W. A.; Coehoorn, R.; Bobbert, P. A. Monte Carlo Study of Charge Transport in Organic Sandwich-Type Single-Carrier Devices: Effects of Coulomb Interactions. Phys. Rev. B 2011, 83, 085206.

(10) Jakobsson, M.; Stafstrom, S. Polaron Effects and Electric Field Dependence of the Charge Carrier Mobility in Conjugated Polymers. J. Chem. Phys. 2011, 135, 134902.

(11) Mendels, D.; Tessler, N. Drift and Diffusion in Disordered Organic Semiconductors: The Role of Charge Density and Charge Energy Transport. J. Phys. Chem. C 2013, 117, 3287-3293.

(12) Watkins, P. K.; Walker, A. B.; Verschoor, G. L. B. Dynamical Monte Carlo Modelling of Organic Solar Cells: The Dependence of Internal Quantum Efficiency on Morphology. Nano Lett. 2005, 5, 1814-1818.

(13) Marsh, R. A.; Groves, C.; Greenham, N. C. A Microscopic Model for the Behavior of Nanostructured Organic Photovoltaic Devices. J. Appl. Phys. 2007, 101, 083509.

(14) Deibel, C.; Strobel, T.; Dyakonov, V. Origin of the Efficient Polaron-Pair Dissociation in Polymer-Fullerene Blends. Phys. Rev. Lett. 2009, 103, 036402.

(15) Meng, L. Y.; Shang, Y.; Li, Q. K.; Li, Y. F.; Zhan, X. W.; Shuai, Z. G.; Kimber, R. G. E.; Walker, A. B. Dynamic Monte Carlo Simulation for Highly Efficient Polymer Blend Photovoltaics. J. Phys. Chem. B 2010, 114, 36-41.

(16) Groves, C. Developing Understanding of Organic Photovoltaic Devices: Kinetic Monte Carlo Models of Geminate and Non-Geminate Recombination, Charge Transport and Charge Extraction. Energ Environ Sci 2013, 6, 3202-3217.

(17) Heiber, M. C.; Baumbach, C.; Dyakonov, V.; Deibel, C. Encounter-Limited ChargeCarrier Recombination in Phase-Separated Organic Semiconductor Blends. Phys. Rev. Lett. 2015, 114, 136602.

(18) Mesta, M.; Carvelli, M.; de Vries, R. J.; van Eersel, H.; van der Holst, J. J. M.; Schober, M.; Furno, M.; Lüssem, B.; Leo, K.; Loebl, P.; Coehoorn, R.; Bobbert, P. A. Molecular-Scale 
Simulation of Electroluminescence in a Multilayer White Organic Light-Emitting Diode. Nat. Mater. 2013, 12, 652-658.

(19) Coehoorn, R.; van Eersel, H.; Bobbert, P.; Janssen, R. Kinetic Monte Carlo Study of the Sensitivity of OLED Efficiency and Lifetime to Materials Parameters. Adv. Funct. Mater. 2015, 25, 2024-2037.

(20) van Eersel, H.; Bobbert, P. A.; Janssen, R. A. J.; Coehoorn, R. Monte Carlo Study of Efficiency Roll-Off of Phosphorescent Organic Light-Emitting Diodes: Evidence for Dominant Role of Triplet-Polaron Quenching. Appl. Phys. Lett. 2014, 105, 143303.

(21) Shen, Y. F.; Giebink, N. C. Monte Carlo Simulations of Nanoscale Electrical Inhomogeneity in Organic Light-Emitting Diodes and Its Impact on Their Efficiency and Lifetime. Phys. Rev. Appl. 2015, 4, 054017.

(22) Demeyu, L.; Stafstroem, S.; Bekele, M. Monte Carlo Simulations of Charge Carrier Mobility in Semiconducting Polymer Field-Effect Transistors. Phys. Rev. B 2007, 76, 155202.

(23) Kwiatkowski, J. J.; Frost, J. M.; Nelson, J. The Effect of Morphology on Electron FieldEffect Mobility in Disordered $C_{60}$ Thin Films. Nano Lett. 2009, 9, 1085-1090.

(24) Sharma, A.; van Oost, F. W. A.; Kemerink, M.; Bobbert, P. A. Dimensionality of Charge Transport in Organic Field-Effect Transistors. Phys. Rev. B 2012, 85, 235302.

(25) Wang, W.; Li, L.; Ji, Z.; Lu, N.; Lu, C.; Xu, G.; Liu, M. In Monte Carlo Simulation of the Dynamic Charge Hopping Transport in Organic Thin Film Transistors, International Conference on Simulation of Semiconductor Processes and Devices, Washington, D.C., 9-11 Sept. 2015; pp 132-135.

(26) Li, H. Y.; Li, Y.; Li, H.; Bredas, J.-L. Organic Field-Effect Transistors: A 3D Kinetic Monte Carlo Simulation of the Current Characteristics in Micrometer-Sized Devices. Adv. Funct. Mater. 2017, 27, 1605715.

(27) Coropceanu, V.; Cornil, J.; da Silva, D. A.; Olivier, Y.; Silbey, R.; Bredas, J. L. Charge Transport in Organic Semiconductors. Chem. Rev. 2007, 107, 926-952.

(28) Tessler, N.; Preezant, Y.; Rappaport, N.; Roichman, Y. Charge Transport in Disordered Organic Materials and Its Relevance to Thin-Film Devices: A Tutorial Review. Adv. Mater. 2009, 21, 2741-2761.

(29) Miller, A.; Abrahams, E. Impurity Conduction at Low Concentrations. Phys. Rev. 1960, $120,745-755$. 
(30) Marcus, R. A. Electron-Transfer Reactions in Chemistry - Theory and Experiment. Rev Mod Phys 1993, 65, 599-610.

(31) Meng, L. Y.; Wang, D.; Li, Q. K.; Yi, Y. P.; Bredas, J. L.; Shuai, Z. G. An Improved Dynamic Monte Carlo Model Coupled with Poisson Equation to Simulate the Performance of Organic Photovoltaic Devices. J. Chem. Phys. 2011, 134, 124102.

(32) Simmons, J. G. Generalized Formula for the Electric Tunnel Effect between Similar Electrodes Separated by a Thin Insulating Film. J. Appl. Phys. 1963, 34, 1793-1803.

(33) Simmons, J. G. Potential Barriers and Emission - Limited Current Flow between Closely Spaced Parallel Metal Electrodes. J. Appl. Phys. 1964, 35, 2472-2481.

(34) Li, H. Y.; Duan, L.; Zhang, D. Q.; Qiu, Y. Electric Field inside a Hole-Only Device and Insights into Space-Charge-Limited Current Measurement for Organic Semiconductors. J. Phys. Chem. C 2014, 118, 9990-9995.

(35) Li, H. Y.; Qiu, Y.; Duan, L. Multi-Scale Calculation of the Electric Properties of Organic-Based Devices from the Molecular Structure. Org. Electron. 2016, 33, 164-171.

(36) Li, H. Y.; Li, C.; Duan, L.; Qiu, Y. Charge Transport in Amorphous Organic Semiconductors: Effects of Disorder, Carrier Density, Traps, and Scatters. Isr. J. Chem. 2014, 54, 918-926.

(37) Ullah, M.; Fishchuk, I. I.; Kadashchuk, A.; Stadler, P.; Pivrikas, A.; Simbrunner, C.; Poroshin, V. N.; Sariciftci, N. S.; Sitter, H. Dependence of Meyer-Neldel Energy on Energetic Disorder in Organic Field Effect Transistors. Appl. Phys. Lett. 2010, 96, 213306.

(38) Kimber, R. G. E.; Wright, E. N.; O'Kane, S. E. J.; Walker, A. B.; Blakesley, J. C. Mesoscopic Kinetic Monte Carlo Modeling of Organic Photovoltaic Device Characteristics. Phys. Rev. B 2012, 86, 235206.

(39) Tamboli, M. S.; Palei, P. K.; Patil, S. S.; Kulkarni, M. V.; Maldar, N. N.; Kale, B. B. Polymethyl Methacrylate (PMMA)-Bismuth Ferrite (BFO) Nanocomposite: Low Loss and High Dielectric Constant Materials with Perceptible Magnetic Properties. Dalton Trans. 2014, 43, 13232-13241.

(40) Preezant, Y.; Roichman, Y.; Tessler, N. Amorphous Organic Devices - Degenerate Semiconductors. J. Phys.-Condes. Matter 2002, 14, 9913-9924.

(41) van der Holst, J. J. M.; Uijttewaal, M. A.; Balasubramanian, R.; Coehoorn, R.; Bobbert, P. A.; de Wijs, G. A.; de Groot, R. A. Modeling and Analysis of the Three-Dimensional Current 
Density in Sandwich-Type Single-Carrier Devices of Disordered Organic Semiconductors. Phys. Rev. B 2009, 79, 085203. 


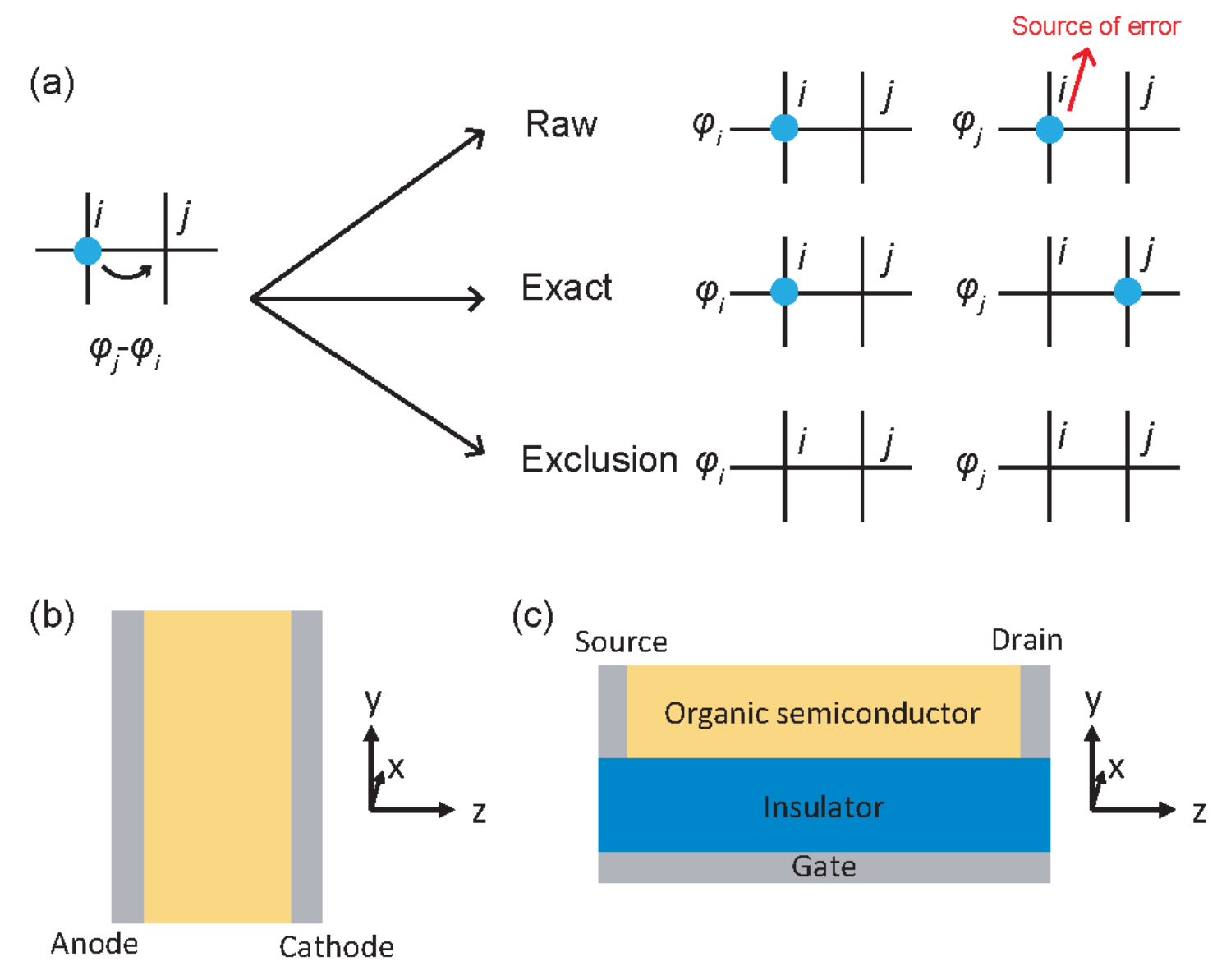

Figure 1. (a) Illustration of the carrier occupations used to determine the electric potentials at sites $i$ and $j$ in the "raw", "exact", and "exclusion" methods; blue spheres represent charge carriers. Illustration of (b) the diode device and (c) the bottom-gate bottom-contact OFET device simulated in this work. 


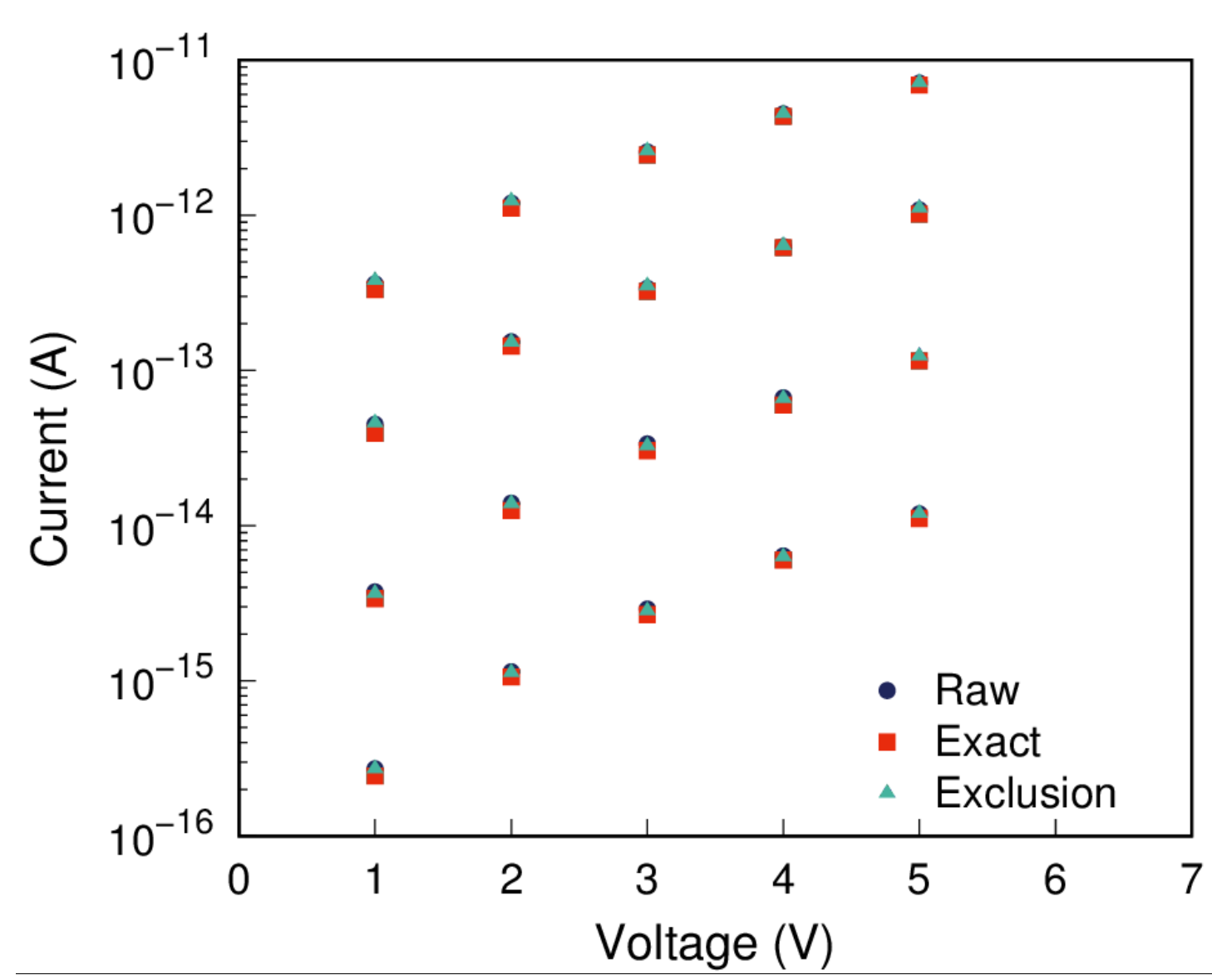

Figure 2. Current vs. voltage for the simulated diode device as a function of energetic disorder $(\sigma)$ for the three KMC methodologies. 

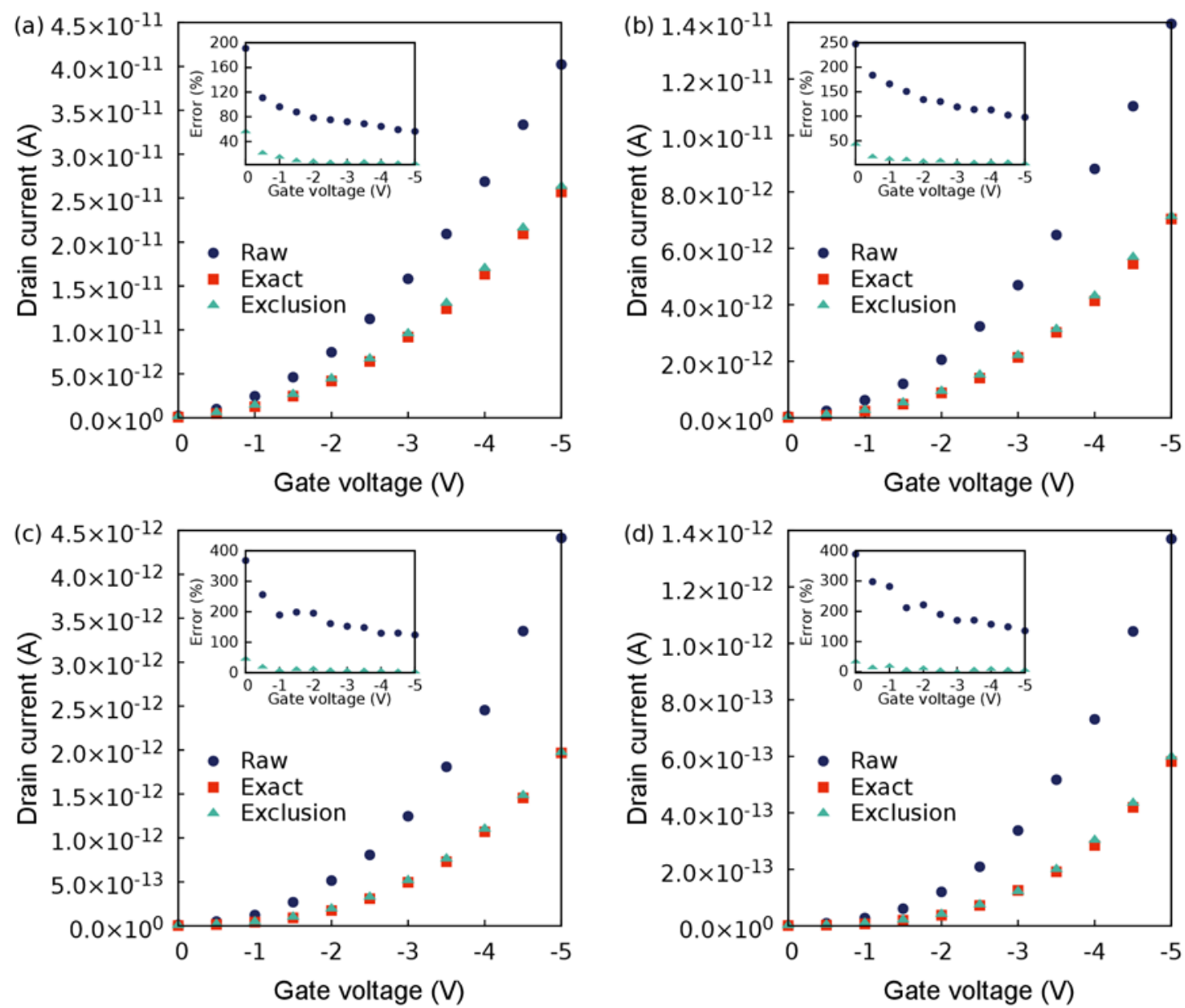

Figure 3. Calculated transfer currents for the OFET device at energetic disorder of: (a) $51 \mathrm{meV}$; (b) $77 \mathrm{meV}$; (c) $103 \mathrm{meV}$; and (d) $128 \mathrm{meV}$. The insets show the errors of the "raw" method and the "exclusion" method relative to the "exact" method. The drain voltage is $-5 \mathrm{~V}$. 

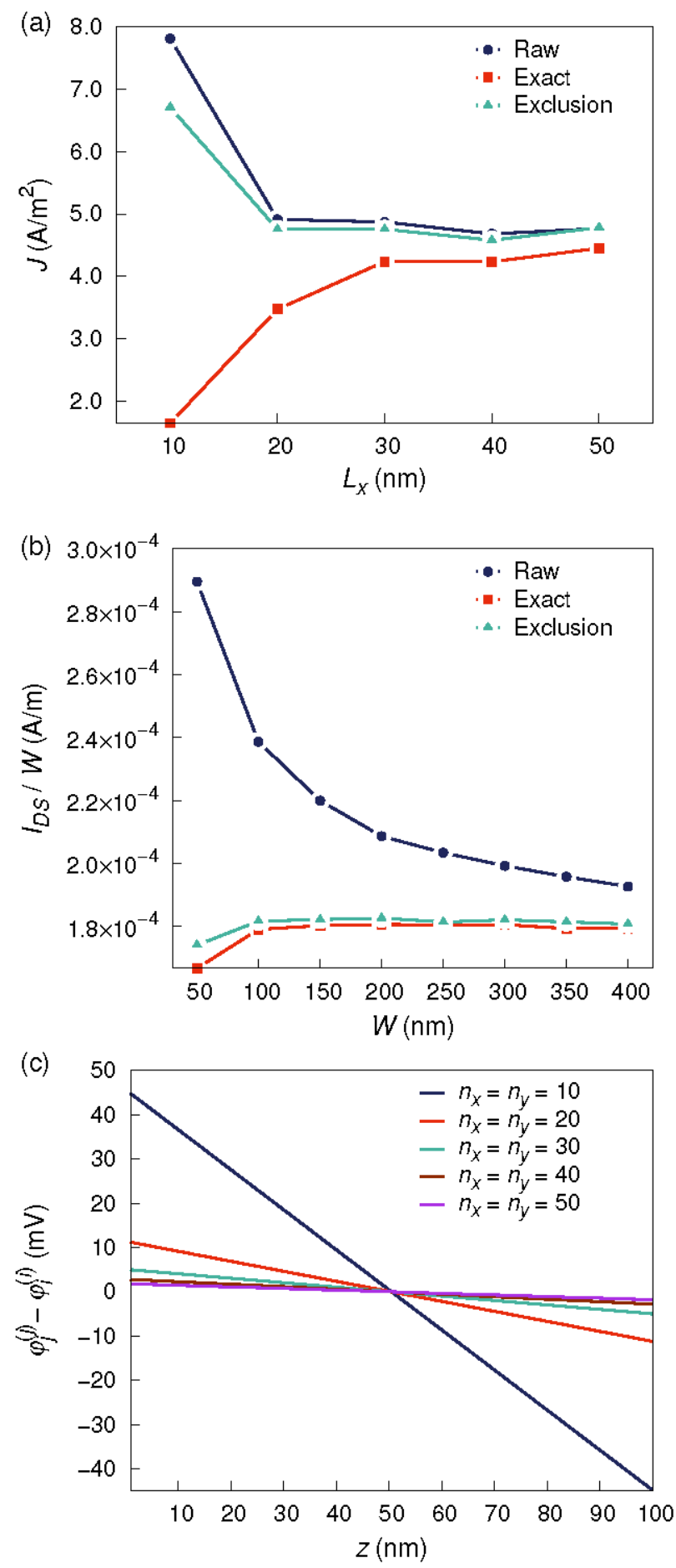

Figure 4. (a) Calculated current densities $(J)$ of diode devices of different dimensions $\left(L_{y}=L_{x}\right.$ in all cases) for $\sigma=128 \mathrm{meV}$ and $V=-5 \mathrm{~V}$; (b) calculated current ( $\left.I_{\mathrm{DS}}\right)$ as a function of width of the OFET device $W$ for $\sigma=51 \mathrm{meV}$ and gate and drain voltages $=-3 \mathrm{~V}$; (c) difference between $\varphi_{j}{ }^{(j)}$ and $\varphi_{i}{ }^{(i)}$ at different positions in the diode device; site $j$ is the site adjacent to site $i$ along the direction of the electric field. 